\title{
EFFECTS OF THE HARMONIC SEXTUPOLE ON TRANSVERSE MULTIBUNCH INSTABILITIES AT ELETTRA
}

\author{
$\underline{\text { L. Tosi }}^{\#}$ and E. Karantzoulis, Sincrotrone Trieste, Trieste, Italy
}

\begin{abstract}
The results of measurements on the ELETTRA storage ring which show a strong dependence of the excitation of transverse multibunch instabilities on the settings of the harmonic sextupole are presented. A strong correlation with the coefficients involved in the non-linear tune shifts with amplitude induced by the sextupole has been observed.
\end{abstract}

\section{INTRODUCTION}

In order to achieve an emittance of $7.0 \mathrm{~nm}$ rad at $2.0 \mathrm{GeV}$, the lattice of ELETTRA was designed to be a double bend achromat of the expanded Chasman Green type with strong focussing quadrupoles. The resulting fairly large natural chromaticities are compensated by two sextupole families in the dispersive arcs. In addition, a family of harmonic sextupoles has been introduced in the dispersion free straight sections, in order to enlarge the dynamic aperture and thus compensate to a large extent the nonlinear effects of the chromatic ones.

In general, non-linear elements produce a distorsion of the phase space distribution of the particles, accompanied by a non-linear tune shift with amplitude, so that two particles with different amplitudes will oscillate with different tunes. For sextupoles, the tune shifts with amplitude appear as higher order effects and are obtainable by applying perturbation theory to the Hamiltonian describing the dynamics in the action and angle variables [1]. For the purpose of this paper, only the tune shifts with amplitude due to second order effects of the sextupoles will be considered. These may be expressed according to :

$$
\begin{aligned}
& \Delta v_{\mathrm{x}}=\mathrm{C}_{11} 2 \mathrm{~J}_{\mathrm{x}}+\mathrm{C}_{12} 2 \mathrm{~J}_{\mathrm{y}} \\
& \Delta \mathrm{v}_{\mathrm{y}}=\mathrm{C}_{12} 2 \mathrm{~J}_{\mathrm{x}}+\mathrm{C}_{22} 2 \mathrm{~J}_{\mathrm{y}}
\end{aligned}
$$

where the subscripts $\mathrm{x}$ and $\mathrm{y}$ denote the horizontal and vertical planes respectively, $\Delta \mathrm{v}_{\mathrm{u}}$ are the non-linear tune shifts, $\mathrm{J}_{\mathrm{u}}$ the perturbed action variables and $\mathrm{C}_{11}, \mathrm{C}_{12}, \mathrm{C}_{22}$ are coefficients which depend on the sextupole strengths, on the beta functions at their locations and on the relative phase advances between them [1,2]. Figure 1 shows the theoretical behaviour of the three coefficients as functions of the harmonic sextupole settings ( nominal value is 46 A).

It has been observed in ELETTRA that the harmonic sextupole S1 plays an essential role in the dynamics when

\footnotetext{
\#Email: tosi@elettra.trieste.it
}

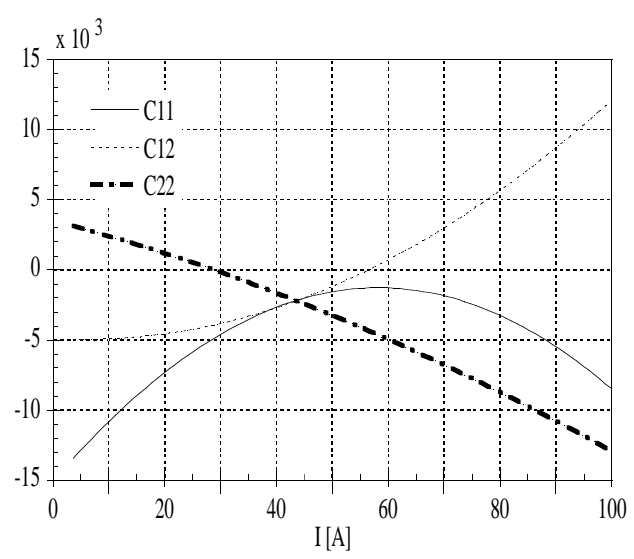

Figure 1: Theoretical behaviour of the three coefficients $\mathrm{C}_{11}, \mathrm{C}_{12}$ and $\mathrm{C}_{22}$ as functions of the harmonic sextupole settings.

the beam is being affected by coherent transverse multibunch instabilities, to the extent that they can even be totally sup-pressed for appropriate settings of the sextupole [3]. Many measurements were performed and they all indicate a strong correlation with the behaviour of the coefficients $\mathrm{C}_{11}$ and $\mathrm{C}_{22}$. This paper presents the main results of the observations, of the measurements performed and of preliminary simulations.

\section{MEASUREMENTS}

Modes driven by the four rf cavities in ELETTRA are controlled by setting the temperatures of the cavities [4]. Most of the measurements were made in the presence of horizontal coupled bunch modes (HCBM), in that it was found quite difficult to excite vertical cavity driven modes in the range of temperatures at which the rf cavities routinely operate. In particular, the behaviour of HCBM 318 with the harmonic sextupole settings, driven by the cavity HOM T3 [5], was investigated, as well as that of HCBM 414-417 the origin of which is still a matter of investigation. Since the horizontal instabilities are rather strong, with visibly large oscillations observed on the synchrotron light beam profile monitor, measurements concentrated on correlating the behaviour of the modes with $\mathrm{C}_{11}$.

Figure 2 compares $\mathrm{C}_{11}$ with the amplitude of HCBM 318 as a function of the current setting of the harmonic sextupole. The mode could be totally suppressed by setting the sextu-pole below $28 \mathrm{~A}$ or above $90 \mathrm{~A}$. The same parabolic beha viour as $\mathrm{C}_{11}$ was found for the excita- 


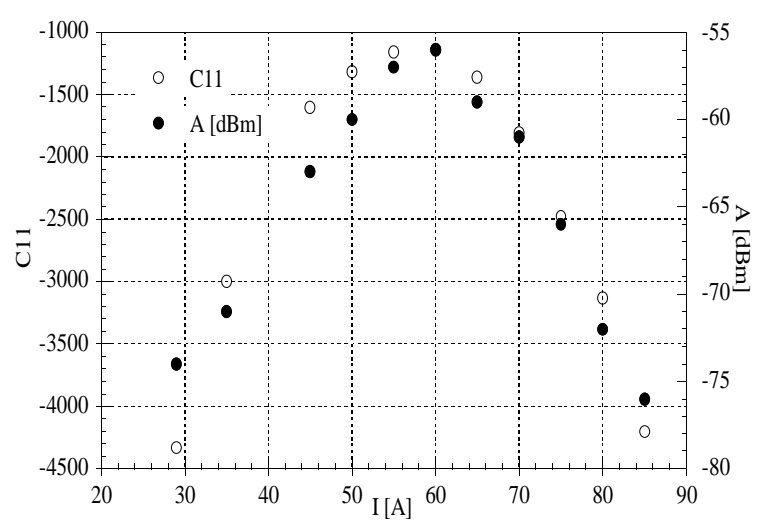

Figure 2: Comparison of the amplitude of HCBM 318 with $\mathrm{C}_{11}$ as functions of the harmonic sextupole settings

tion levels of HCBM 414, although in this case the two current values of the sextupole for which the mode was totally suppressed were found to be different. While the peak of the excitation level of the modes was always found to be at the setting of $60 \mathrm{~A}$, investigations revealed that the two boundary current values of the sextupole at which the modes can be suppressed depend on the beam current, on the modes excited, on the filling pattern and on the presence or less of longitudinal instabilities. It was noticed that the stronger the mode, the higher the filling percentage and the lower the longitudinal excitations were, the wider was the range of the sextupole settings for which the modes were excited. This namely translates into requiring higher absolute values for $\mathrm{C}_{11}$ in order to achieve the suppression of the horizontal modes. While the dependence on the beam current and on the particular mode excited reflects the 'strength' of the driving force, the one on the filling pattern and on the presence or not of longitudinal modes is thought to be due to how 'well' the driving force manages to couple to the beam's modes. The coupling efficiency of a transverse driving force may be diminished by a gap within the bunch train, by the different arrival times of the bunches and possibly also on the density of the bunches.

It has to be strongly underlined that even in the total absence of longitudinal modes, confirmed by measurements of the full longitudinal spectrum, it was found that the amplitudes of the excited transverse modes follow the same trend of $\mathrm{C}_{11}$ with the harmonic sextupole settings, excluding thus any interference of longitudinal modes in the mechanism by which the sextupole may be influencing the dynamics. This was a particularly important point to confirm, also because the cavities are located in the dispersive arc. Furthermore, it was found, by measuring full longitudinal spectra as a function of the harmonic sextupole settings, that the excitation level of any longitudinal instabilities did not depend on the sextupole.
The major beam parameters, such as tunes, closed orbit, dispersion and chromaticities were measured as a function of the harmonic sextupole settings. No significant changes were noticed in these quantities, with the exception of the horizontal chromaticity, which for the measurement of figure 2 was found to vary from 0.1 for the setting of $\mathrm{I}=28$ A to 1.6 for $\mathrm{I}=90 \mathrm{~A}$ (both currents are values for which the mode was suppressed). This effect is not expected to be the major cause for the suppression of the mode. In fact, no change in the excitation level of the mode was noticed when the horizontal chromaticity was set to 1.6 , by using the chromaticity correcting sextupoles. Since the value of $\mathrm{C}_{11}$ also depends on the settings of the chromaticity correcting sextupoles, investigations were carried out in order to confirm the leading role of $\mathrm{C}_{11}$ in the phenomenon against that of the horizontal chromaticity. The results revealed that the suppression of excited modes using the chromaticity correcting sextupoles required horizontal chromaticities which could be even greater than 7 times the one measured when the suppression was achieved using the harmonic sextupole. The values of $\mathrm{C}_{11}$ instead in the two situations of suppression of the modes were computed to be almost identical.

Low frequencies in the spectrum (below $100 \mathrm{~Hz}$ ) have also been observed, associated with the presence of the transverse modes. Whereas in some situations there appears a well defined peak at low frequency, in others the whole background noise level increases by 1-2 orders of magnitude. Whenever well defined low frequencies were noticed, measurements on the spectrum analyzer in zero span mode of the unstable sideband showed periodicities corresponding to the peaks measured in the low frequency spectrum range. Detailed investigations in a narrow frequency range around the unstable sideband with an appropriate sweeping time showed a fine structure indicating that the sideband was moving with the measured low frequency. It is believed that in this scenario the mode is shifting along the impedance experiencing different real and immaginary parts of it, until it eventually goes out of its frequency range of influence. The beam then loses coherence and is damped until the coherence builds up again. Increasing the absolute value of $\mathrm{C}_{11}$ by lowering the sextupole current changed the dynamics and the unstable sideband presented only a frequency spread. It has been found that the settings of the harmonic sextupole influence the values of the low frequencies. Figure 3 shows the dependence of the low frequencies both on the harmonic sextupole settings and on the beam current for a fixed setting of the sextupole of $60 \mathrm{~A}$. The low frequencies fit extremely well to a second order polynomial as a function of the sextupole, presenting a maximum where the absolute value of $\mathrm{C}_{11}$ is minimum. 


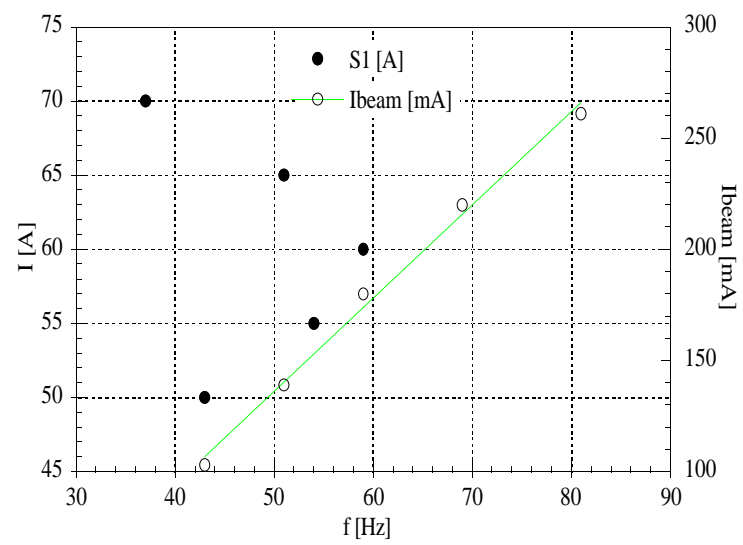

Figure 3: Low frequencies associated to transverse instabilities as functions of the harmonic sextupole settings and of beam current for a fixed setting ( $\mathrm{I}=60 \mathrm{~A})$

Another measurement that was considered necessary was to confirm that effectively the horizontal tune shift with amplitude $\Delta v_{\mathrm{x}}$ follows the same behaviour as $\mathrm{C}_{11}$. For this purpose, the horizontal tune spread of a $1 \mathrm{~mA}$ beam in single bunch mode was measured as a function of the sextupole settings, by exciting the beam with a constant amplitude using the spectrum analyser of the tune measurement system. A good agreement was found.

Table 1: Amplitudes of vertical modes vs. harmonic sextupole settings

\begin{tabular}{|c|c|c|c|c|}
\hline $\begin{array}{c}\text { Setting } \\
{[\mathrm{A}]}\end{array}$ & $\begin{array}{c}\text { VCBM } \\
167[\mathrm{~dB}]\end{array}$ & $\begin{array}{c}\text { VCBM } \\
246[\mathrm{~dB}]\end{array}$ & $\begin{array}{c}\text { VCBM } \\
344[\mathrm{~dB}]\end{array}$ & $\begin{array}{c}\text { VCBM } \\
422[\mathrm{~dB}]\end{array}$ \\
\hline 30 & 16.7 & 16.3 & 17.0 & 37.3 \\
\hline 36 & 18.6 & 10.8 & 11.0 & 38.7 \\
\hline 91 & 6.1 & - & - & 25.4 \\
\hline
\end{tabular}

Only recently, it was possible to find temperature settings of the cavities which would give rise to vertical modes. Assuming the same hypothesis as for the horizontal plane, some preliminary studies have been made on the dependence of the vertically excited modes on $\mathrm{C}_{22}$. As it can be seen in figure $1, \mathrm{C}_{22}$ presents very low values, in the range of settings for the harmonic sextupole between 20 and $40 \mathrm{~A}$, becoming fairly large for settings above 60 A. Thus, if the hypothesis that the coefficients in the tune shift with amplitude is the leading mechanism by which excitation levels of the instabilities depend on the harmonic sextupole, one would expect an enhancement of the vertical modes for lower settings of the sextupole and suppression for higher ones. Table 1 illustrates the amplitudes of the modes as function of the settings. In particular, during the measurement, it was noticed that trying to set the harmonic sextupole to $27 \mathrm{~A}\left(\mathrm{C}_{22}=0\right.$ for $26 \mathrm{~A} \mathrm{)}$, the vertical instability grew stronger with an evident vertical beam size blow up on the synchrotron light beam profile monitor and subsequent loss of 100 $\mathrm{mA}$ of beam current.

\section{CONCLUSIONS}

All measurements which have been performed indicate that there is a strong correlation between the levels of excitation of transverse coupled bunch instabilities and the coefficients involved in the non-linear tune shift with amplitude induced by sextupoles. Theoretical investigations with simulations of the cavity transverse HOMs are being undertaken in parallel on both the effect of the harmonic sextupole on the internal distributions of amplitudes within a bunch and its effect on the dynamics when the bunches are considered as macroparticles. Particular attention is being paid on the non-linear tune shift with amplitude, which has a straightforward dependence on the coefficients $\mathrm{C}_{11}$ and $\mathrm{C}_{22}$. One of the hypothesis which is being considered for the phenomenon is that of an induced tune spread among the macroparticles. A tracking program, used also for the design of the transverse feedback system[6], which takes into account sextupoles, HOMs in the cavities and the radiation damping, has been developed. The first preliminary results in the horizontal plane yield a confirmation of the dependence of the excited modes on the harmonic sextupole settings. Amplitudes of particles are found to grow until a certain final level, around which the amplitudes of the particles then perform oscillations. Both the final level and the amplitudes of the oscillations performed once the latter is reached are found to increase as the harmonic sextupole is set to give smaller absolute values of $\mathrm{C}_{11}$, indicating effectively that the mode becomes more excited when $\mathrm{C}_{11}$ is smaller in absolute value, as observed in the measurements.

\section{REFERENCES}

[1] R. Nagaoka et al, "Nonlinear dynamics with sextupoles in lowemittance light source storage rings", Nuclear Instruments and Methods in Physics Research A302 (1991) 9-26

[2] Y. Ng, Fermilab Report TM-1281 (1984)

[3] E. Karantzoulis et al, "Collective Effects at Elettra", EPAC Conference Proceedings, Stockholm, 1998

[4] M. Svandrlik et al., "The Cure of Multibunch Instabilities in Elettra”, PAC Conference Proceedings, Dallas, 1995

[5] M. Svandrlik et al., "Simulations and Measurements of Higher Order Modes of the Elettra RF Cavities in View of Coupled Bunch Instability Compensation by Temperature Variation", EPAC Conference Proceedings, Stiges, 1996

[6] D. Bulfone et all., "Design Considerations for the Elettra Transverse Multibunch Feedback", this conference 